\title{
Análise da utilização da plataforma Khan Academy para a educação matemática
}

\author{
Ynêssa Beatriz Dantas de Farias Santos'섯 Apuena Vieira Gomes ${ }^{1}$ \\ ${ }^{1}$ Instituto Metrópole Digital - Universidade Federal do Rio Grande do Norte (UFRN) \\ 59.078-970 - Natal - RN - Brasil \\ \{ynessafarias@gmail.com, apuena@imd.ufrn.br\}
}

\begin{abstract}
The integration of digital technologies in education associated with mathematics teaching is everyday stronger. The Khan Academy class platform is an example of the many tools that can be used for this purpose. In order to analyze details about how it has been used to improve mathematical education, this paper proposes to investigate, through a Systematic Review, information about its use. With positive results in all the studies analyzed, the use of Khan Academy is feasible and has the potential to simultaneously achieve the integration of digital technologies in education and the improvement in mathematical teaching strategies.
\end{abstract}

Resumo. A integração de tecnologias digitais na educação associada ao ensino da matemática é uma crescente cada vez mais forte. A plataforma de aulas Khan Academy é um exemplo das muitas ferramentas que podem ser usadas com esse objetivo. Com fins de analisar detalhes sobre como ela vem sendo utilizada para melhorar a educação matemática, este artigo propõe investigar, por meio de revisão sistemática da literatura, informações sobre seu uso. Com resultados positivos em todos os estudos analisados, o uso da Khan Academy mostra-se viável e com potencial de alcançar simultaneamente a integração de tecnologias digitais na educação e a melhoria nas estratégias de ensino da matemática.

\section{Introdução}

A adoção de tecnologias digitais da informação e comunicação (TDIC) tem ganhado cada vez mais força em todas as áreas da sociedade. Pode-se afirmar que "a informática compõe hoje a ecologia cognitiva na qual todos nós estamos inseridos", não sendo mais possível conceber contextos educativos desvinculados dessa realidade [Oliveira, Costa e Moreira 2001, p. 60]. Sendo a educação a mola propulsora do desenvolvimento social, as tecnologias digitais não poderiam estar fora dos recursos que a compõem. $\mathrm{O}$ uso de TDICs é um fator primordial para a oferta de uma educação de qualidade e conectada com as demandas da sociedade moderna. A presença de ferramentas digitais nas instituições de ensino, associada a um uso crítico e orientado, potencializa a eficácia da educação, já que as TDIC são parte do nosso cotidiano e influenciam a estruturação do pensamento, modo de ser, agir e pensar das gerações que hoje frequentam nossas salas de aula [Almeida e Valente 2011, p. 06]. 
Esse uso, ao passar por mudanças, perdeu o espaço que um dia foi reservado para as ultrapassadas "aulas de informática", nas quais os alunos aprendiam exclusivamente o passo-a-passo mecânico sobre a utilização de alguns programas e aplicativos, e ganhou contextualização e envolvimento ao ser integrado às disciplinas tradicionais [Valente 2000]. Ao invés de aprender a operar o computador isoladamente, os alunos têm aulas de português, por exemplo, em plataformas ou aplicativos, e aprendem a operar a máquina ao mesmo tempo em que recebem os conteúdos regulares da disciplina. Um movimento integrado e completamente associado às tarefas do dia-adia, como o é fora dos espaços escolares, exigindo "uma nova visão de educação, condizente com a acelerada mudança que estamos presenciando em praticamente todos os segmentos da sociedade" [Almeida e Valente 2011, p. 70].

Uma das escolhas para essa integração pode facilmente ser a matemática. De acordo com documentos de análise e comparação a nível internacional, publicados pela Organização para a Cooperação e Desenvolvimento Econômico (OCDE), o Brasil está entre os dez países com pior rendimento escolar em matemática, ciências e leitura, no Programa Internacional de Avaliação de Estudantes (Pisa) - Programme for International Student Assessment [OCDE 2016]. Segundo o mesmo estudo, 67,1\% dos alunos brasileiros apresentam um baixo rendimento e proficiência em matemática. Já conforme o Education at a glance, publicado pela OCDE em 2017, o Brasil possui uma das porcentagens mais baixas ( $17 \%$ comparados a $24 \%$ da média dos países analisados) de estudantes formados nas áreas de ciência, tecnologia, engenharia e matemática (STEM na sigla em inglês), o que pode ser resultado de experiências de insucesso na educação das ciências exatas ao longo da educação básica.

Diante desse cenário, e como parte dos estudos que compõem uma dissertação de mestrado na área, pesquisou-se sobre os resultados da aplicação de uma plataforma digital para o ensino da matemática na educação básica. A plataforma Khan Academy (KA) foi fundada em 2006 e possui o nome do seu idealizador, Salman Khan. Teve início quando Khan começou a gravar vídeos para auxiliar uma sobrinha a superar dificuldades em matemática. As gravações foram colocadas em um site de streaming de vídeos e se tornaram um sucesso entre estudantes que buscavam materiais para reforçar seus estudos [KHAN 2013]. Hoje a Khan Academy dispõe de cada vez mais recursos, como cursos gratuitos em várias áreas, possibilidade de monitoramento e mapeamento do desempenho dos alunos. Sabendo que a ferramenta em questão se encaixa nos conceitos de Wiley (2008) e Batista et al (2017), para Objeto de Aprendizagem (OA), quais sejam, materiais reutilizáveis, com características didáticopedagógica e que possam ser direcionados a conteúdos específicos; e sabendo também que a ferramenta atende às demandas atuais para a inserção de tecnologias digitais na educação, o estudo foi conduzido priorizando buscar os resultados já coletados envolvendo o uso da Khan Academy na educação matemática.

O objetivo desta revisão sistemática da literatura (RSL) foi identificar artigos que abordem o uso da plataforma Khan Academy para a melhoria do ensino da matemática na Educação Básica. A principal contribuição deste estudo é unir os resultados em um só documento e apontar os possíveis caminhos com potencial de serem explorados, no que concerne ao uso da plataforma. O texto está dividido em seções, como segue: resultados de outros estudos realizados na área, metodologia 
VIII Congresso Brasileiro de Informática na Educação (CBIE 2019)

Anais do XXV Workshop de Informática na Escola (WIE 2019)

utilizada para a produção da revisão, discussão dos resultados e considerações finais.

\section{Trabalhos relacionados}

As buscas foram feitas nos seguintes bancos de dados: Workshops do $\mathrm{CBIE}^{1}$, $\mathrm{WIE}^{2}$ e $\mathrm{Ctrl}+\mathrm{E}^{3}$, o periódico Renote, com foco na mesma temática, o portal de periódicos da Capes $^{4}$, o Google Acadêmico, além de um evento específico na área de matemática ${ }^{5}$. Dentro das pesquisas feitas não foram encontradas revisões sistemáticas da literatura investigando o uso exclusivo da plataforma Khan Academy. Outros trabalhos, em contrapartida, trazem contribuições acerca do que vem sendo analisado envolvendo o uso de tecnologias digitais na educação. O trabalho de Vieira e Santolini (2018) apresenta uma revisão sobre o uso de tecnologias em currículos escolares. Trata-se de uma revisão bibliográfica sistemática da literatura que teve como objetivo analisar os indícios a respeito da inserção de tecnologias em diferentes aspectos das práticas educacionais no ensino público de educação básica do Brasil, com enfoque em escolas com currículos inovadores. Os autores analisaram oito artigos, bem como os dados de 178 escolas consideradas inovadoras ou em processo de inovação, cadastradas em uma plataforma do MEC. Em suas considerações, o artigo mostrou que há poucas publicações sobre escolas inovadoras no Brasil e o papel da tecnologia nesses ambientes educacionais. Os resultados encontrados mostram que há poucas pesquisas intervencionistas com enfoque prático divulgadas na academia, além de as pesquisas não apresentarem evidências de práticas de integração tecnológica em currículos considerados inovadores.

Foi analisado também o trabalho de Melo, Costa e Maia (2017), sobre recursos educativos digitais para educação matemática, visto que a plataforma que estamos investigando se enquadra como um desses recursos. Os autores se propuseram a registrar o processo de busca, catalogação e classificação (com base nos temas da Prova Brasil) dos recursos educativos digitais para dispositivos móveis incluídos no repositório OBAMA ${ }^{6}$. A busca se deu nos principais repositórios de apps disponíveis e resultou na catalogação de 184 ferramentas, das quais mais de $80 \%$ se encontravam disponíveis na plataforma Google Play Store. A pesquisa de abordagem mista, com enfoques quantitativos e qualitativos, concluiu que há diversos recursos educativos digitais para dispositivos móveis que podem ser usados para potencializar as aulas de matemática. No entanto, alguns desses apps, mesmo estando classificados dentro da área da Matemática, quando submetidos aos parâmetros de avaliação e classificação, não se enquadram para o trabalho em nenhum tema de conteúdo específico da disciplina. Os autores ainda demonstram melhor o processo de análise das ferramentas apresentando detalhes de alguns OA analisados, mas o app da Khan Academy não está entre eles. Ao final do artigo, apesar de sabermos que há um trabalho de catalogação e análise de ferramentas digitais para a educação matemática, não é possível conferir se a Khan Academy está sob observação e/ou pesquisa. Desta forma, reforça-se a importância desta RSL, visto que ela trata exclusivamente da análise do que vem sendo

\footnotetext{
${ }^{1}$ Anais dos Workshops do Congresso Brasileiro de Informática na Educação

${ }^{2}$ Anais do Workshop de Informática na Escola

${ }^{3}$ Anais do Congresso sobre Tecnologias na Educação

${ }^{4}$ Portal de periódicos da Coordenação de Aperfeiçoamento de Pessoal de Nível Superior

${ }^{5}$ Congresso Internacional de Ensino da Matemática

${ }^{6}$ Objetos de Aprendizagem para Matemática - projeto vinculado ao Instituto metrópole Digital, da UFRN
} 
VIII Congresso Brasileiro de Informática na Educação (CBIE 2019)

Anais do XXV Workshop de Informática na Escola (WIE 2019)

verificado quanto à utilização da Khan Academy para fins do ensino da matemática.

\section{Metodologia}

Trata-se de uma revisão sistemática da literatura que compõe a "contextualização teórica do problema e o seu relacionamento com o que tem sido investigado a seu respeito", esclarecendo os pressupostos teóricos que fundamentam a pesquisa e as contribuições geradas pelas investigações anteriores [Gil 2002, p. 162]. De acordo com orientações do Centre for Reviews and Dissemination (2008), as quais serviram de inspiração para o passo-a-passo desta RSL, os principais estágios que constituem um trabalho de revisão da literatura são os seguintes: I) O protocolo da revisão: 1) Áreaschave para cobrir em um protocolo de revisão (onde foram incluídas as questões de pesquisa); 2) Aprovação do projeto de protocolo; 3) Possíveis alterações do protocolo durante a revisão. II) Realização da revisão: 1) Identificação de evidências de pesquisa para revisão sistemática (onde foram definidas as bases de dados consultadas); 2) Seleção dos estudos (com a aplicação dos critérios de inclusão e exclusão); 3) Extração dos dados; 4) Avaliação da qualidade; 5) Síntese dos dados; 6) Relatório escrito; 7) Registro da revisão; 8) Divulgação dos resultados.

Esta RSL utilizou as seguintes questões de pesquisa (QP): QP1.: Em que níveis de ensino a plataforma Khan Academy tem sido mais explorada? QP2.: A plataforma tem sido usada como recurso para as aulas regulares ou como atividade extracurricular? QP3.: Que conteúdos de matemática disponibilizados na plataforma são utilizados?

\subsection{Bases de dados consultadas e estratégias de busca}

As bases de dados selecionadas são uma mescla dos anais dos principais eventos na área de tecnologias na educação, quais sejam: Workshops do CBIE, WIE e Ctrl+E, o periódico Renote, o portal de periódicos da Capes, o Google Acadêmico, além dos anais do Congresso Internacional do Ensino da Matemática. Essas bases foram escolhidas por possuírem grande parte das publicações sobre Informática na Educação no Brasil e devido ao acesso livre e gratuito ao seu material. As pesquisas foram conduzidas no mês de junho de 2019 e priorizaram as publicações produzidas nos últimos cinco anos. A primeira etapa da pesquisa teve foco na leitura dos títulos dos trabalhos e a consulta se deu sob duas strings de busca, a depender da configuração da base consultada, a saber: uma utilizando o termo "Khan Academy" e outra apenas com o termo "matemática or mathematics", esta para quando o título não contivesse o termo Khan Academy, mas a abordagem direta com a matemática pudesse trazer o uso da plataforma ao longo do artigo; resultados nos quais foi feita também a leitura do resumo. Os termos foram digitados no campo de busca de cada base e, em seguida, os arquivos reportados foram separados para, então, terem seus títulos analisados. A tabela 01 contém os resultados da primeira busca, com os arquivos apresentados após a string ser inserida no campo de busca.

O total de 185 artigos reportados passou por refinamento em duas fases, dentro de critérios de inclusão e exclusão, antes de serem submetidos à leitura completa. Os trabalhos reportados após a busca pela string "Matemática or Math", passaram pela leitura do resumo para que pudéssemos conferir se algum deles explorava o uso da Khan Academy para a educação matemática, mesmo não havendo menção da 
VIII Congresso Brasileiro de Informática na Educação (CBIE 2019)

Anais do XXV Workshop de Informática na Escola (WIE 2019)

plataforma no título do artigo. Além disso, foi feita uma busca automática por termochave no corpo do artigo, buscando por "Khan Academy", a fim de garantir se havia algum trecho do artigo que fosse interessante para a presente revisão. Do total de 120 artigos nessa condição, nenhum deles apresentou menção ao uso da plataforma, nem no resumo, nem na busca automática por termo-chave. Os temas centrais desses trabalhos giram em torno de apps, jogos, pensamento computacional, recursos educativos digitais, repositórios de OA, ambientes educacionais, formação docente, robótica e avaliação da aprendizagem, todos voltados à educação matemática.

Tabela 01: Resultados das strings de busca por base de dados

\begin{tabular}{|c|c|c|c|c|c|c|c|}
\hline \multirow[t]{2}{*}{ Base } & \multirow[t]{2}{*}{ String de busca } & \multicolumn{5}{|c|}{$\begin{array}{c}\text { Anos pesquisados/ estudos } \\
\text { encontrados }\end{array}$} & \multirow[t]{2}{*}{ Total } \\
\hline & & 2018 & 2017 & 2016 & 2015 & 2014 & \\
\hline \multirow{2}{*}{$\begin{array}{c}\text { Anais do Workshop } \\
\text { do CBIE }\end{array}$} & Khan Academy & 0 & 0 & 0 & 0 & 0 & 0 \\
\hline & Matemática or Math & 14 & 6 & 7 & 6 & 4 & 37 \\
\hline \multirow{2}{*}{ Anais do WIE } & Khan Academy & 1 & 1 & 0 & 0 & 0 & 2 \\
\hline & Matemática or Math & 5 & 13 & 4 & 4 & 6 & 32 \\
\hline \multirow{2}{*}{$\mathrm{Ctrl}+\mathrm{E}$} & Khan Academy & 0 & 0 & 1 & - & - & 1 \\
\hline & Matemática or Math & 6 & 9 & 3 & - & - & 18 \\
\hline \multirow{2}{*}{ Revista Renote } & Khan Academy & 0 & 0 & 0 & 1 & 0 & 1 \\
\hline & Matemática or Math & 7 & 7 & 6 & 8 & 5 & 33 \\
\hline $\begin{array}{c}\text { Congresso Internacional } \\
\text { de Ensino da } \\
\text { Matemática (CIEM) }\end{array}$ & Khan Academy & - & 1 & - & - & - & 1 \\
\hline Portal Capes & Khan Academy & \multicolumn{5}{|c|}{52 (nos últimos cinco anos) } & 52 \\
\hline Google Acadêmico & $\begin{array}{c}\text { Khan Academy AND (Math } \\
\text { or Mathematics or } \\
\text { Matemática) }\end{array}$ & \multicolumn{5}{|c|}{8 (nos último cinco anos) } & 8 \\
\hline \multicolumn{7}{|c|}{ Total de estudos } & 185 \\
\hline
\end{tabular}

Fonte: elaborada pelos autoras

Para os 65 resultados restantes, foram aplicados os seguintes critérios de inclusão (CI): CI1: Trabalhos devem ser revisados por pares; CI2: Trabalhos escritos em português ou inglês; CI3: Menção do uso da plataforma Khan Academy no título, associado ao ensino e aprendizagem; CI4: Trabalhos primários.

Ainda dentro dessa etapa de afunilamento dos resultados, foram aplicados os seguintes critérios de exclusão (CE): CE1: Trabalhos repetidos - resultados que apareceram em mais de uma base de dados; CE2: Trabalhos envolvendo o uso da plataforma Khan Academy para outros fins que não a educação matemática; CE3: Trabalhos incompletos; CE4: Títulos indisponíveis para consulta. A tabela 2 demonstra os resultados obtidos após a aplicação desses critérios.

\subsection{Critérios de qualidade dos estudos}

Os 17 estudos restantes (vide listagem) após a aplicação dos critérios de inclusão e exclusão passaram por leitura completa e foram submetidos a critérios de qualidade para, então, serem relatados nesta revisão. É necessário deixar claro que os critérios estabelecidos mantêm relação com o fato de as questões de pesquisa terem, ou não, 
VIII Congresso Brasileiro de Informática na Educação (CBIE 2019)

Anais do XXV Workshop de Informática na Escola (WIE 2019)

sido respondidas após a leitura completa dos trabalhos. Caso o artigo não trate claramente dos pontos específicos que foram investigados, isso não quer dizer que ele seja um trabalho de baixa qualidade; quer dizer, tão somente, que não foi suficientemente útil para os fins desta RSL. Dessa forma, foram determinados os seguintes critérios de qualidade (CQ): CQ1: O trabalho responde às questões de pesquisa? CQ2: O estudo traz contribuições aos problemas descritos na introdução desta RSL? CQ3: A relevância da pesquisa está claramente identificada? CQ4: A temática está devidamente fundamentada teoricamente? CQ5: O estudo traz informações suficientes para situar o leitor no contexto no qual foi produzido? CQ6: Os métodos apresentados no estudo podem ser replicados em outras pesquisas?

A cada estudo submetido a esses critérios foram dadas as respostas "Sim" (representada pelo símbolo $\checkmark$ ); "Não" (representada por X) ou "Parcialmente" (representada por $(-)$ ), conforme demonstrado na tabela 03 .

Tabela 02: Filtragem dos resultados pelos critérios de inclusão e exclusão

\begin{tabular}{|c|c|c|c|c|c|c|c|c|c|c|}
\hline Base & $\begin{array}{c}\text { Estudos } \\
\text { encontrados }\end{array}$ & $\begin{array}{c}\text { Após } \\
\text { CI1 }\end{array}$ & $\begin{array}{r}\text { Após } \\
\text { CI2 }\end{array}$ & $\begin{array}{r}\text { Após } \\
\text { CI3 }\end{array}$ & $\begin{array}{r}\text { Após } \\
\text { CI4 }\end{array}$ & $\begin{array}{r}\text { Após } \\
\text { CE1 }\end{array}$ & $\begin{array}{r}\text { Após } \\
\text { CE2 }\end{array}$ & $\begin{array}{c}\text { Após } \\
\text { CE3 }\end{array}$ & $\begin{array}{r}\text { Após } \\
\text { CE4 }\end{array}$ & Total \\
\hline $\begin{array}{c}\text { Anais do } \\
\text { WIE }\end{array}$ & 2 & 2 & 2 & 2 & 2 & 2 & 1 & 1 & 1 & 1 \\
\hline Ctrl+E & 1 & 1 & 1 & 1 & 1 & 1 & 0 & 0 & 0 & 0 \\
\hline $\begin{array}{c}\text { Revista } \\
\text { Renote }\end{array}$ & 1 & 1 & 1 & 1 & 1 & 1 & 1 & 1 & 1 & 1 \\
\hline CIEM & 1 & 1 & 1 & 1 & 1 & 1 & 1 & 1 & 1 & 1 \\
\hline Portal Capes & 52 & 24 & 22 & 19 & 19 & 15 & 9 & 9 & 8 & 8 \\
\hline $\begin{array}{c}\text { Google } \\
\text { Acadêmico }\end{array}$ & 8 & 8 & 7 & 7 & 7 & 7 & 7 & 7 & 6 & 6 \\
\hline \multicolumn{7}{|c|}{ Total de estudos } \\
\hline
\end{tabular}

Fonte: elaborada pelos autores

\section{Análise dos estudos selecionados}

Apesar de apenas dois estudos (06 e 12) terem preenchido de forma inteiramente positiva os critérios de qualidade, os demais trabalhos também serão contemplados nesta análise, já que possuem contribuições para uma, ou mais, das questões de pesquisa. Em relação à QP1, sobre os níveis de ensino nos quais a KA tem sido mais explorada, oito estudos $(07,08,09,10,11,13,14,15)$ procuram soluções a nível de educação básica e sete a nível de educação superior (01, 02, 03, 04, 05, 06, 12). Dentre os que trabalham com o nível superior de ensino, o estudo 02 trata especificamente de formação continuada para professores de matemática e os estudos 01, 04, 05 e 06 pretendem investigar maneiras de auxiliar alunos de graduação que não possuem nível de matemática adequado ao curso pretendido na universidade.

Em relação a isso, foi encontrado um dado que merece mais atenção. Ao início do artigo foram apresentados, como justificativa para a pesquisa, dois pontos fundamentais: um que diz respeito à inserção de tecnologias digitais na educação e outro que demonstra a necessidade de melhorar as aulas e recursos de aprendizagem da 
VIII Congresso Brasileiro de Informática na Educação (CBIE 2019)

Anais do XXV Workshop de Informática na Escola (WIE 2019)

matemática no Brasil, dados os baixos resultados nas avaliações de desempenho a nível nacional e internacional. O mapa representado na figura 01 mostra em destaque os países nos quais os estudos analisados ocorreram.

Tabela 03: Análise dos estudos conforme critérios de qualidade

\begin{tabular}{|c|c|c|c|c|c|c|}
\hline Estudo & CQ1 & CQ2 & CQ3 & CQ4 & CQ5 & CQ6 \\
\hline 01 & $\checkmark$ & $\checkmark$ & $(-)$ & $\checkmark$ & $\checkmark$ & $\checkmark$ \\
\hline 02 & $(-)$ & $\checkmark$ & $\checkmark$ & $\checkmark$ & $\checkmark$ & $\checkmark$ \\
\hline 03 & $\checkmark$ & $\checkmark$ & $\checkmark$ & $\checkmark$ & $\checkmark$ & $(-)$ \\
\hline 04 & $\checkmark$ & $(-)$ & $(-)$ & $\checkmark$ & $(-)$ & $(-)$ \\
\hline 05 & $\checkmark$ & $\checkmark$ & $\checkmark$ & $\checkmark$ & $\checkmark$ & $(-)$ \\
\hline 06 & $\checkmark$ & $\checkmark$ & $\checkmark$ & $\checkmark$ & $\checkmark$ & $\checkmark$ \\
\hline 07 & $\checkmark$ & $\checkmark$ & $(-)$ & $\checkmark$ & $\checkmark$ & $\checkmark$ \\
\hline 08 & $(-)$ & $\checkmark$ & $\checkmark$ & $\checkmark$ & $\checkmark$ & $\checkmark$ \\
\hline 09 & $(-)$ & $X$ & $\checkmark$ & $\checkmark$ & $\checkmark$ & $\checkmark$ \\
\hline 10 & $\checkmark$ & $(-)$ & $\checkmark$ & $\checkmark$ & $\checkmark$ & $(-)$ \\
\hline 11 & $(-)$ & $\checkmark$ & $\checkmark$ & $\checkmark$ & $\checkmark$ & $(-)$ \\
\hline 12 & $\checkmark$ & $\checkmark$ & $\checkmark$ & $\checkmark$ & $\checkmark$ & $\checkmark$ \\
\hline 13 & $(-)$ & $\checkmark$ & $\checkmark$ & $\checkmark$ & $\checkmark$ & $\checkmark$ \\
\hline 14 & $(-)$ & $X$ & $\checkmark$ & $\checkmark$ & $(-)$ & $(-)$ \\
\hline 15 & $\checkmark$ & $(-)$ & $\checkmark$ & $\checkmark$ & $\checkmark$ & $(-)$ \\
\hline 16 & $(-)$ & $\checkmark$ & $\checkmark$ & $(-)$ & $\checkmark$ & $(-)$ \\
\hline 17 & $(-)$ & $\checkmark$ & $\checkmark$ & $\checkmark$ & $\checkmark$ & $(-)$ \\
\hline
\end{tabular}

Fonte: elaborada pelos autores

Figura 01: Distribuição geográfica da origem dos estudos analisados

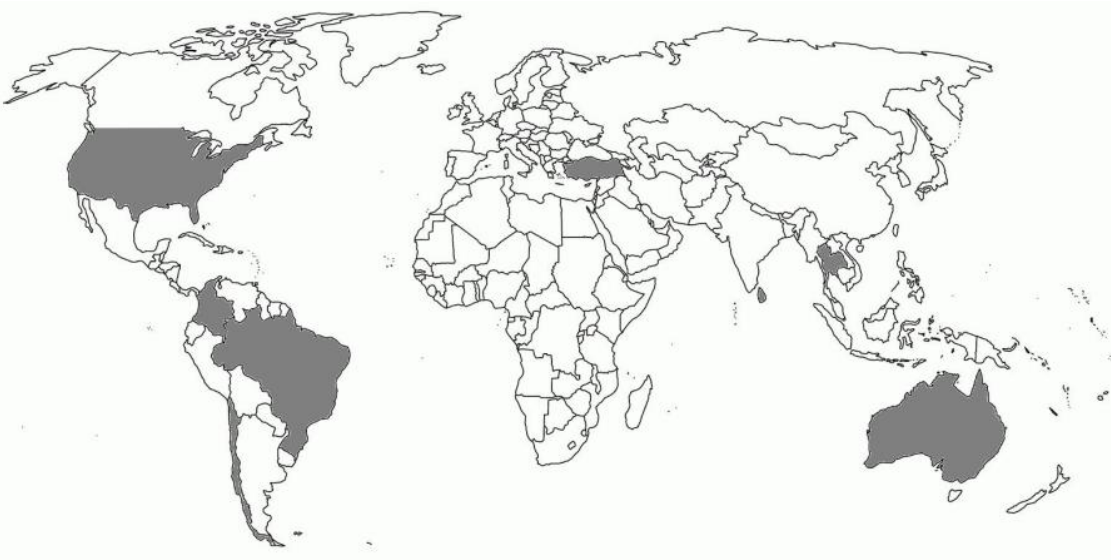

Fonte: elaborada pelos autores

A curiosidade aqui é o fato de todos esses países enfrentarem, em algum nível, 
VIII Congresso Brasileiro de Informática na Educação (CBIE 2019)

Anais do XXV Workshop de Informática na Escola (WIE 2019)

a mesma problemática registrada pelos brasileiros em relação à aprendizagem matemática. Os estudos $02,03,10,12,14$ e 15 dão um foco maior à questão da inserção de tecnologias digitais no contexto educacional e à adequação aos meios tecnológicos modernos com fins suprir as demandas da educação nos dias atuais. Já os demais estudos, todos eles, mencionam a necessidade de melhorar os resultados na aprendizagem matemática de um modo geral. É possível considerar, pois, que as dificuldades no ensino da matemática não são exclusivas do Brasil, mas trata-se de uma questão a ser melhorada a nível global.

A QP2 interroga se o estudo traz informações sobre se a plataforma Khan Academy vem sendo/foi usada como recurso para as aulas regulares ou como atividade complementar. Quanto a isso, os estudos 02, 04, 05, 08 e 09 relatam o uso da KA como recurso das aulas regulares. Com exceção do estudo 15, que menciona o uso da plataforma como atividade regular e complementar para melhoria do aprendizado dos alunos, todos os demais estudos mencionam o uso da plataforma de maneira complementar. Dentre os 17 estudos analisados, a KA é uma das ferramentas mais utilizadas quando se trata ou se aplicam metodologias do ensino híbrido. Alguns estudos, como é o caso dos 03, 06 e 12 fazem menção à metodologia já no seu título. Os demais reforçam a adoção do ensino híbrido em seu aporte teórico e como uma possibilidade de integrar tecnologias digitais ao ensino. A esse respeito, o trabalho 14 menciona que o ensino híbrido tem sido cada vez mais adotado, e com resultados positivos, para as séries da educação básica.

Já a QP3 trouxe a dúvida sobre quais conteúdos da Matemática foram mais escolhidos dentre os disponibilizados na plataforma. Alguns estudos são meramente de cunho investigativo sobre se a utilização da KA demonstra evidências de sucesso entre os usuários ou sobre quais ferramentas digitais têm sido mais escolhidas para o estudo da matemática, como é o caso dos estudos 11 e 14. Os estudos 01, 08, 09, 10, 13, 14, 16 e 17, por sua vez, não fazem menção a que conteúdos foram explorados, mas mencionam que foram apropriados para a série ou nível dos alunos, ou que a experiência permitiu que os usuários escolhessem livremente o que gostariam de explorar. Alguns estudos, em contrapartida, mencionam especificamente o conteúdo escolhido, como segue: artigos 03 e 04: Funções e Variações, Pré-álgebra e álgebra; artigo 05: Cálculo Diferencial; artigos 06 e 12: Integrais Duplas; artigos 07 e 15: Geometria. O artigo 02, que trata de formação continuada para professores de matemática, teve como assunto principal a exploração da plataforma, já que o públicoalvo não conhecia a KA.

Um ponto em comum na análise dos 17 estudos selecionados são os resultados positivos mencionados. O grupo, ou os grupos de alunos investigados em cada artigo apresentaram melhorias no desempenho quantitativo ou qualitativo na matemática. Os estudos 01, 03 e 04, por exemplo, tratam de experiências de cursos de apoio ao início da vida acadêmica, para alunos que não iniciaram os estudos de graduação com o nível ideal de aprendizagem na matemática. Há menção de excelentes resultados nas notas desses alunos e diminuição dos níveis de retenção. $O$ estudo 17, por sua vez, traz dados qualitativos sobre os níveis de ansiedade dos alunos em relação às provas de matemática. Foi verificado aumento da autoestima, uma vez que os alunos passaram a aprender melhor os conceitos estudados e, por causa disso, consequente diminuição da ansiedade. O estudo 13 deixa claro que os membros da equipe escolar não concordam se o aumento da perseverança dos alunos se deu pelo 
VIII Congresso Brasileiro de Informática na Educação (CBIE 2019)

Anais do XXV Workshop de Informática na Escola (WIE 2019)

uso da plataforma e, concordando com o que os estudos 15 e 16 trouxeram, defendem que há inúmeros fatores trabalhando juntos no impacto do aumento de desempenho dos alunos e o uso da plataforma é um deles.

\section{Considerações Finais}

Este trabalho teve como objetivo investigar o uso específico da plataforma Khan Academy na educação matemática. Após sequência do protocolo de pesquisa, resultaram para a análise 17 artigos, dos quais foram retirados dados que respondessem às questões de pesquisa. Foi possível notar que há pouco material registrado sobre a KA nos principais eventos brasileiros que integram tecnologias digitais e educação. Também foi possível perceber que a plataforma vem sendo usada para suprir demandas tanto dos níveis de ensino básico como superior e que suas características, como o acesso ilimitado e gratuito, por exemplo, têm garantido que países de todo o mundo a utilizem como recurso educativo digital. Isso também permitiu verificar que em todas essas localidades as dificuldades em relação aos processos de ensino e aprendizagem da matemática se repetem, incluindo países ditos do primeiro mundo. Os resultados positivos quanto à utilização da plataforma nos 17 estudos analisados mostram que a utilização desse recurso tem potencial de suprir diversas dificuldades na educação matemática, desde que devidamente monitorada por profissionais da área e apoiada por demais membros da comunidade escolar.

\section{Referências}

Almeida, M. E. B. de; Valente, J. A. (2011). "Tecnologias e currículo: trajetórias convergentes ou divergentes? ", Paulus - (Coleção Questões Fundamentais da Educação - 10), São Paulo.

Gil, A, C. (2002). "Como elaborar projetos de pesquisa”, Atlas, São Paulo.

Khan, Salman. (2013) "Um mundo, uma escola: a educação reinventada”, Intrínseca. Rio de Janeiro.

Melo, E. M.; Costa, C. J. N.; Maia, D. L. (2017). Recursos educativos digitais para Educação Matemática: um levantamento para dispositivos móveis. In: Congresso sobre Tecnologias na Educação, 2017, Mamanguape/PB. (Anais do Ctrl+E 2017), p. $916-925$.

OCDE. (2016). “Alunos de baixo desempenho: por que ficam para trás e como ajudálos? “, Paris: OECD Publishing.

Oliveira, C. C.; Costa, J. W. da; Moreira, M. (2001). “Ambientes informatizados de aprendizagem: produção e avaliação de software educativo", 1ed. Papirus, (Coleção Prática Pedagógica), Campinas/SP.

Centre for Reviews and Dissemination. (2009). "Systematic Reviews. CRD's guidance for undertaking reviews in health care", https://bit.ly/2Q0pjBy, Junho, 2019.

Valente, J.A. (Org.) (2000). “O computador na sociedade do conhecimento”. MEC, Brasília.

Wiley, D. A. (2008). "Connecting learning objects to instructional design theory: a definition, a metaphor, and a taxonomy". Logan: Utah State University. 